\title{
Feasibility Study of Time-of-Flight Compton Scatter Imaging Using Picosecond Length X-ray Pulses.
}

\author{
Nick Calvert, Marta M. Betcke, Alick N. Deacon, Anthony J. Gleeson, Clive Hill, Peter A. McIntosh, \\ Lawrence O. Mitchell, Edward J. Morton, James Ollier, Mark G. Procter, and Robert D. Speller
}

\begin{abstract}
By measuring the Time-of-Flight (ToF) of scattered $x$-ray photons, the point of interaction, assuming a single scatter, can be determined, providing three dimensional information about an object under inspection. The present work describes experimental ToF Compton scatter measurements conducted at The Versatile Electron Linear Accelerator (VELA), a picosecond pulse width electron source situated in Daresbury, UK. The ToF of scattered $\mathrm{x}$-ray photons was measured using a CeBr3 detector, and a Full Width at Half Maximum (FWHM) of between $29 \mathrm{~cm}$ and $36 \mathrm{~cm}$ was achieved with a $5 \mathrm{~cm}$ thick plastic test object. By implementing a low energy cut off, the FWHM was reduced to between $12 \mathrm{~cm}$ and $26 \mathrm{~cm}$. Two test objects placed in series with a $50 \mathrm{~cm}$ space between were separable in the data after applying the low energy cut off.
\end{abstract} rity.

Index Terms-X-ray scattering, Time-of-Flight, Cargo Secu-

\section{INTRODUCTION}

$\mathbf{T}$ HE ability to image objects in three dimensions is highly beneficial in security screening as it simplifies image analysis by reducing the number of complex, overlapping objects that can be present in two dimensional images. Using transmission x-ray imaging, this can be achieved using either tomography or tomosynthesis. These require the source and detectors to rotate, either physically or electronically, around the object under inspection. Alternatively the detector and source can be kept in a constant position and the object can be rotated. Both of these methods have been realised for the screening of airport baggage [1], [2], however, three dimensional imaging of cargo containers provides greater challenges. Both tomography and tomosynthesis are infeasible due to the large size of the containers, and any such system would require a long screening time, and have a high cost [3].

Conventional methods of radiation-based screening rely on transmission imaging, where contrast is provided by the attenuation of photons as they pass through objects, or Compton Scatter Imaging (CSI), where contrast is provided by photons scattering off objects. Three dimensional CSI is possible, however it typically exploits the Compton scatter energy-angle relationship, requiring the use of $\gamma$-ray sources [4] which are typically lower flux than electronic x-ray sources, and are constantly 'on'. Alternatively, three dimensional CSI can be

Manuscript received May 27, 2014; revised August 1, 2014.

N. Calvert, MM. Betcke, and RD. Speller are with University College London, London, UK. E-mail: n.calvert.11@ucl.ac.uk.

LO. Mitchell, AN. Deacon, J. Ollier, MG. Procter, and EJ. Morton are with Rapiscan Systems Cargo Division, Stoke-on-Trent, UK.

AJ Gleeson, C Hill and PA. McIntosh is with Science and Technologies Facilities Council, Daresbury, UK. performed using x-ray sources [5], however this requires the use of extensive collimation which is photon inefficient.

An alternative method is to measure the Time-of-Flight (ToF) of photons scattering off an object under inspection. The ToF of photons can be used to recover the point of interaction and provide three dimensional information about the object. The aim of the present experimental studies is to examine the feasibility of using $\mathrm{ToF}$ measurements to recover interaction points in the third dimension, and whether this can ultimately result in three dimensional images in future experiments. This article provides a brief description of the concepts involved, before outlining the initial proof of principle experiments, undertaken at The Versatile Electron Linear Accelerator (VELA) at the Science and Technologies Facilities Council, Daresbury, the data analysis methods used and finally the results and conclusions.

\section{CONCEPT}

The Compton scatter interaction occurs between an incident $\mathrm{x}$-ray photon and an electron in the scattering material. The photon is deflected through a scattering angle $\theta$, and a portion of its energy is transferred to the electron (called the recoil electron). The energy loss of the photon depends on the initial energy of the photon, $\mathrm{E}_{0}$, and the scatter angle, $\theta$. The relation is given by the following equation [6]

$$
E_{1}\left(E_{0}, \theta\right)=\frac{E_{0}}{1+\alpha(1-\cos \theta)},
$$

where $\alpha=E_{0} / 511 \mathrm{keV}$ is the ratio between the incident photon energy and the rest-mass energy of the electron, and $E_{1}$ is the energy of the scattered photon.

The requirement of access to only one side of the container is one of the advantages of using CSI for cargo security. The necessary scattering angles of the photons to be incident upon the detector is therefore close to $\pi$. This results in a fundamental limit on the energy of the scattered photon:

$$
\lim _{E_{0} \rightarrow+\infty} E_{1}\left(E_{0}, \pi\right)=\frac{\alpha}{2}=255.5 \mathrm{keV}
$$

which in turn limits the depth of penetration it can achieve. For a $4.55 \mathrm{MeV}$ x-ray beam, only $1 \%$ of photons are able to penetrate $20 \mathrm{~cm}$ of plastic before scattering through $\pi \mathrm{rad}$ and penetrating back through the $20 \mathrm{~cm}$ of plastic. CSI is therefore preferred when screening sparsely filled cargo or cars, whereas high-energy, high penetration, transmission imaging is preferred for densely packed cargo. 
ToF imaging using ionising radiation has historically been restricted to applications that use a positron emitting radioactive source. ToF Positron Emission Tomography (PET) has received a high amount of interest recently, with the improvement in detector technology allowing for much greater resolution [7]. Developments have focused on faster photodetectors [8] and scintillators [9]. Recent developments in photodetectors has seen the focus shift from Photomultiplier Tubes (PMTs) to Silicon Photomultiplier Tubes (SiPMs). In particular, the development of the digital SiPM combines the advantages of the SiPM, fast timing resolution, low bias voltage, no magnetic field sensitivity, whilst requiring no external electronic processing [10], [11]. The developments in SiPMs, combined with new fast and bright inorganic scintillators such as LSO:Ce, $\mathrm{LaBr}_{3}$ and $\mathrm{CeBr}_{3}$, has seen sub 100 ps timing resolutions achievable [12]. The downside to the use of SiPMs is their small size. When a large array is needed, a large number of channels is required and the data handling becomes computationally expensive. PMTs have also provided excellent timing resolution, with $154 \mathrm{ps}$ achieved in [13].

Another method of ToF imaging using positron sources is to detect one of the primary $511 \mathrm{keV}$ photons in delayed coincidence with the other primary photon that has undergone a scattering event in an object under inspection. This technique has been applied to industrial measurements [14], landmine detection [15], [16], and soil moisture measurements [17]. The method has also been used to examine objects behind barriers [18], which suggests the technique has possible applications in cargo security imaging, with spatial resolution as low as $5 \mathrm{~cm}$ achievable [19]. The present work differs by using a pulsed x-ray source instead of a positron source. When using a positron source, it is expected that at most only 1 gammaray will scatter back per trigger. When using a high flux $\mathrm{x}$ ray source, there are many $\mathrm{x}$-ray photons per source trigger, which suggests the technique will be more efficient, assuming a sufficiently high trigger rate.

The ToF of scattered x-ray photons can be used to reconstruct the position of interaction if the time distribution of the source is known. The simplest case is to use a source with a Dirac delta distribution time profile. Since such sources do not exist, the next best solution is to use a source with an extremely short pulse width (of the order of picoseconds). Due to the timing resolution of current $\mathrm{x}$-ray detectors being of the order of $100 \mathrm{ps}$ [20], such a source can be considered to have a Dirac delta distribution pulse width. In two dimensions, the region of constant ToF given such a source and a point detector is an ellipse whose foci are the source and detector coordinates. The semi-major and semi-minor axes, $a$ and $b$, respectively, are defined as

$$
\begin{aligned}
2 a & =c t, \\
b^{2} & =a^{2}-f^{2},
\end{aligned}
$$

where $c$ is the speed of light, $t$ is the ToF of the $\mathrm{x}$-ray photon, and $2 f$ is the distance between the source and the detector. This is illustrated in Figure 1. Using a collimated pencil beam of $\mathrm{x}$ rays allows for direct reconstruction of the scattering point, defined by the intersection of the pencil

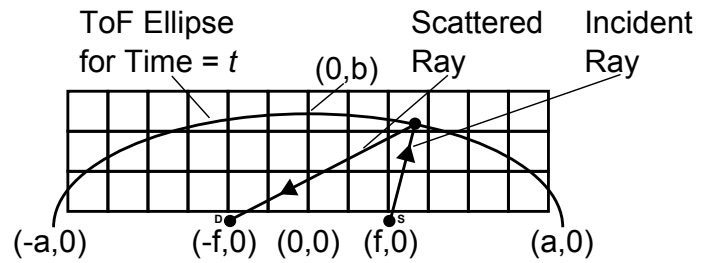

Fig. 1. Points of equal ToF in a pixel grid. The ellipse is centred at the origin, the detector is placed at $(-f, 0)$, and the source at $(f, 0)$.

beam and ellipse. An image can be formed by sweeping such a beam over an object of interest, and recording the ToF of the scattered $x$-ray photons. In principle one could reduce measurement times by using a fan beam and image reconstruction techniques.

In order to achieve sufficient signal-to-noise ratios in the $\mathrm{x}$ ray ToF measurements, a high flux source is required. This results in several x-ray photons incident upon the detector during a very short time period. The resultant voltage from the detector will contain signals from these $\mathrm{x}$-ray photons and will be a pulse pileup event. To maximise the signal-tonoise ratio, these pulse pileup events must be deconvolved into single pulses accurately, which can be performed by assuming the pileup is a combination of single, shifted, pulses and solving the resultant equations to find the time and energy of the pulses [21]. Such deconvolution techniques have been of increased interest recently as pileups have a detrimental effect on energy spectra and the overall quality of a measurement [22]. A number of deconvolution methods have been developed based on different approaches [23][25], the ultimate aim of these methods is to find the energy of each interaction. Although the time of interaction is also found using these methods, it is typically not used. In a ToF application the main aim is to find the time of interaction, and the secondary aim is to find the energy of each deposition.

The accuracy of the deconvolution method will have a large effect on the spatial resolution possible with ToF x-ray measurements, along with the size and timing resolution of the detector, and the size of the pencil beam used. These effects are described in more detail in the subsequent sections of the paper.

The experiments conducted used an extremely short pulse width $\mathrm{x}$-ray source. The technique can still work with wider pulse sources, if the pulse distribution is known, using deconvolution techniques [26]. Using commercially available linear accelerators with known pulse shapes would allow the technique to be used in the field, without the need for expensive, short pulse width $\mathrm{x}$-ray sources.

\section{EXPERIMENTAL SETUP}

\section{A. Versatile Electron Linear Accelerator}

VELA is a modular injector facility capable of providing high quality electron beams for industrial applications, situated at the Science and Technologies Facilities Council, Daresbury [27]. Acceleration is provided by a 2.5-cell, S-band, RF gun resulting in a maximum electron energy of $4.55 \mathrm{MeV}$ [28]. 


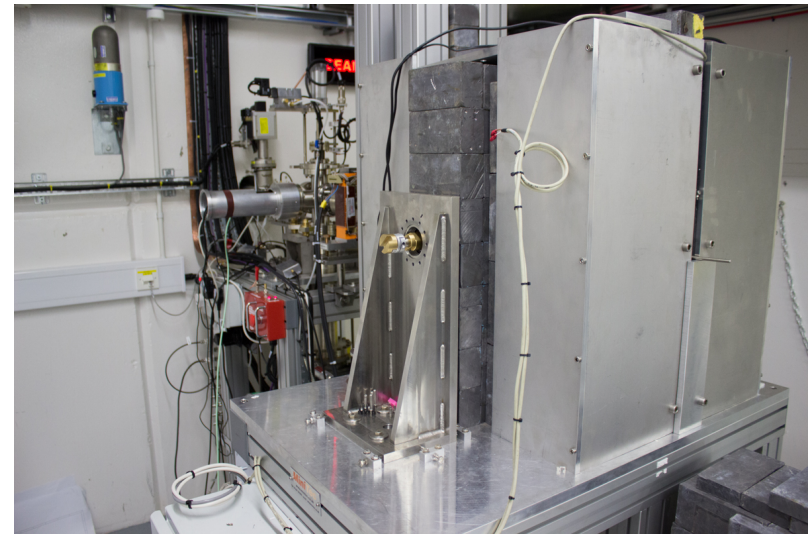

Fig. 2. The x-ray source and collimator situated at VELA. The target is shielded using a $60 \mathrm{~cm} \times 60 \mathrm{~cm} \times 60 \mathrm{~cm}$ lead collimator. The collimator had a $5.5 \mathrm{~mm}$ radius aperture to create a pencil beam of $\mathrm{x}$ rays with an opening angle of approximately $2^{\circ}$.

This is driven by a 76 fs pulse length laser. The electron bunch expands due to space charge effects, estimated to be $1.3 \mathrm{ps}$ at the end of the gun and approximately $4 \mathrm{ps}$ at the user area, in simulation, at $250 \mathrm{pC}$ [29]. The average bunch charge was measured at $100 \mathrm{pC}$, however there was some significant variation over time of the bunch charge, caused by changes in the RF phase. The accelerator operated at $10 \mathrm{~Hz}$, and each data acquisition lasted 5,000 source triggers.

A $0.7 \mathrm{~mm}$ thick, $4.5 \mathrm{~mm}$ radius tungsten target was placed incident to the electron beam to produce $\mathrm{x}$ rays, which were then collimated. A photograph of the collimator is shown in Figure 2.

\section{B. Detector \& Data Acquisition System}

The detector used in the experimental work was a $76 \mathrm{~mm}$ diameter, $19 \mathrm{~mm}$ thick, $\mathrm{CeBr}_{3}$ scintillator attached to an Electron Tubes $9821 \mathrm{~KB}$ linear focused photomultiplier tube, operating at $-1500 \mathrm{~V}$. The photomultiplier tube had both dynode and anode readout, however only the dynode readout was used as the risetime was shorter than the anode readout. A photograph of the detector is given in Figure 4, and the measured risetime and decaytime have been plotted in Figure 3.

$\mathrm{CeBr}_{3}$ is a detector well suited for $\mathrm{ToF}$ measurements due to its short decaytime, high optical photon yield $\left(68,000 \mathrm{MeV}^{-1}\right)$, and low background radioactivity [30]. Intrinsic radioactivity of a scintillator creates noise in the ToF measurements, and hence $\mathrm{CeBr}_{3}$ was preferred to lanthanum and lutetium based detectors which have a higher activity. Using $\left({ }^{N} / \tau_{D}\right)^{-1 / 2}(N$ is the number of optical photons produced, and $\tau_{D}$ is the decay constant of the scintillator) as an indication of timing resolution of a scintillator [31], $\mathrm{LaBr}_{3}$ would have resulted in a timing resolution improvement of approximately $17 \%$, whilst LYSO would result in a decrease of $100 \%$. The intrinsic backgrounds are $1-2$ counts $/ \mathrm{s} / \mathrm{cm}^{3}$ and 250 counts $/ \mathrm{s} / \mathrm{cm}^{3}$ [32], respectively, which could introduce significant noise when using a large array of detectors and a higher pulse rate source.

The detector had a large surface area, to maximise the

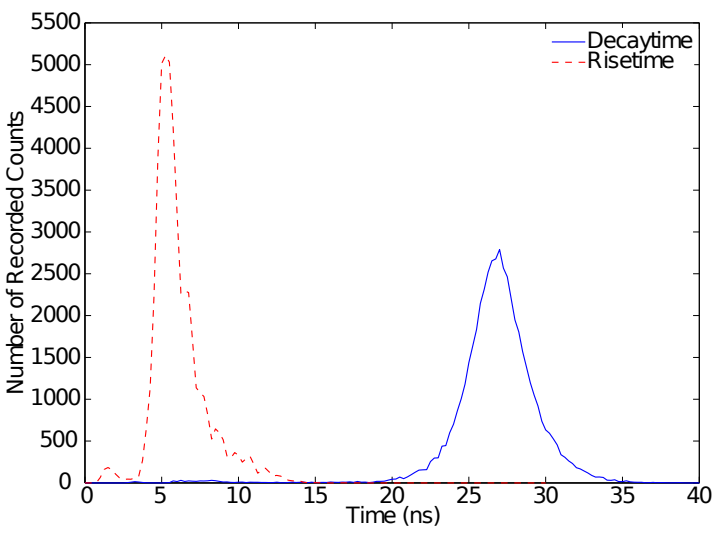

Fig. 3. The measured risetime (dashed) and decaytime (solid) of dynode traces from the $\mathrm{CeBr}_{3}$ detector. The risetime was obtained by measuring the time taken between $10 \%$ to $90 \%$ of pulse height on the rising edge of the detector traces, and the decaytime was calculated by fitting a straight line to the log of the decay curve of each detector trace. The average risetime was $6.1 \mathrm{~ns}$ and the average decaytime was $26.8 \mathrm{~ns}$.

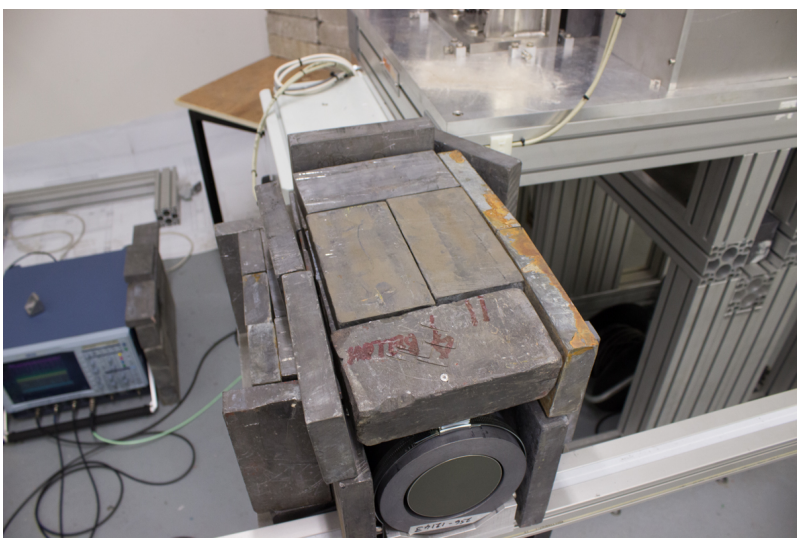

Fig. 4. The detector used for the experiments. $76 \mathrm{~mm}$ thick lead shielding was built up around the detector.

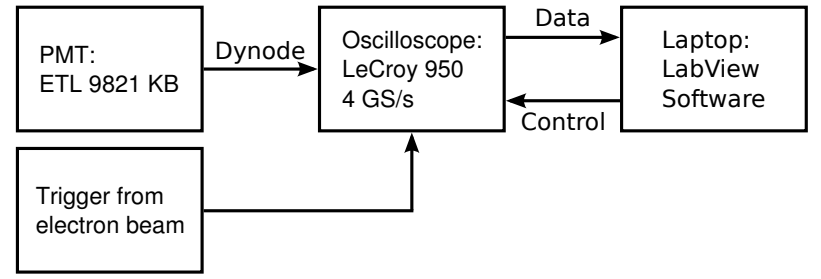

Fig. 5. Schematic of the electronic setup and data flow. Data analysis was done offline, after the data acquisition was completed.

number of x-ray photons detected, and therefore the signalto-noise ratio.

The signals from the detector were recorded using a LeCroy WavePro 950 oscilloscope running at $4 \mathrm{GS} / \mathrm{s}$, which was controlled using a programme written in the LabVIEW [33] software package. Data analysis, described later, was done offline after data collection. A simple schematic of the electronic set up can be found in Figure 5.

\section{Test Objects}


Two experiments were performed. Experiment 1 involved measurements of ToF of photons scattered from a single object (a $5 \mathrm{~cm}$ thick block of high density polyethylene). The object was placed in a beam at three different positions denoted $T 1, T 2$ and $T 3,0.5 \mathrm{~m}$ apart, see Figure 6a. For position T2 measurements were collected for three detector positions marked D1, D2 and D3 in Figure 6a, and for positions T1 and $\mathrm{T} 3$ only at detector position D1 due to the limited time we had the facility available.

In Experiment 2 the photons scattering off three test objects placed in a series were measured, as depicted in Figure 6b. Measurements were taken at detector positions D2 and D3 in order to reduce attenuation, and multiple scatter. The background signal ie. scatter without the object in the beam was measured for all detector positions.

\section{Pulse Pileup Deconvolution}

Due to the large surface area of the detector, and the high flux of the source, it was expected that several photons would be incident upon the detector during each pulse of the x-ray source, resulting in a pulse pileup event. An example of the detector signal from such an event can be seen in Figure 7 (solid line), where a number of separate pulses are visible in the pile up. A single pulse event has been included for comparison.

To maximise the precision of the ToF measurements, the pileup events must be deconvolved into single events accurately. The ToF of the individual $\mathrm{x}$-ray photons in the pileup event can be calculated using the deconvolved, single $x$-ray photon, signals.

The pulse pileup signal, $y_{p p}$, of an ideal detector resulting from $N$ x-ray photons can be written as follows

$$
y_{p p}(t)=\sum_{i=1}^{N} E_{i} y_{s p}\left(t-t_{i}\right),
$$

where $E_{i}$ is the amplitude, and $t_{i}$ is the time of arrival of the $i$ 'th pulse in the pileup event, and $y_{s p}\left(t-t_{i}\right)$ is the single $\mathrm{x}$-ray photon response of the detector, shifted by the time of arrival. All of the amplitudes are strictly greater than 0 , as a photon must deposit energy for it to be detectable.

In reality, detectors are not ideal and the resultant signals must undergo analog-to-digital conversion before they can be recorded. For a digitised signal, the discrete version of Equation 5 reads

$$
y_{p p}^{d}(\hat{t})=\sum_{i=1}^{n} E_{i} y_{s p}^{d}\left(\hat{t}-\hat{t}_{i}\right),
$$

where $n$ is the number of samples in the digitised signal, $y_{p p}^{d}$ and $y_{s p}^{d}$ are the discrete versions of $y_{p p}$ and $y_{s p}$, respectively and with $\hat{t}$ we denote the discrete time sampled every 250 picoseconds (for a $4 \mathrm{GS} / \mathrm{s}$ oscilloscope). If there are $N$ pulses in the pileup event, and $T$ is the set of their time of arrivals, then the following holds

$$
\begin{aligned}
& E_{i}>0, \quad \forall t_{i} \in T, \\
& E_{i}=0, \quad \forall t_{i} \notin T .
\end{aligned}
$$

Assuming the single photon response of the detector is known, Equation 6 can be solved using least squares, with a nonnegativity constraint, giving both the amplitude, and the time of arrival of the photons. For ideal measurements, the accuracy of the computations is limited by the discretization, while electronic and background noise will deteriorate the results.

The single x-ray photon response was obtained by recording 50100 detector traces with no source present. These traces were sorted by pulse height, and an average pulse shape was taken for each possible pulse height. Prior to taking the average, the signals were aligned using a cross correlation method. The first recorded pulse at each pulse height was used as a reference, and the other pulses were shifted until the difference in the rising edge was minimised, an average of the pulses was then taken. Figure 8 shows a plot of the average pulse shape (solid line) and the standard deviation (dashed line) for pulses with an amplitude of $45 \mathrm{mV}$.

The pulse shape plotted in Figure 8 was used for the deconvolution method to find the time of arrival of the x-ray photons. The pulse shape did not significantly change with pulse height, and for simplicity only one pulse height was used to find the mean pulse shape. The average pulse shape for $45 \mathrm{mV}$ amplitude pulses was used for the algorithm as it was the most frequently occurring pulse height when measuring the background, ensuring the greatest certainty in the pulse shape. After deconvolution, it was often found that two consecutive (250 ps apart), low amplitude, pulses fitted the pileup data better than a single high amplitude pulse, even though the data appeared to result from a single pulse only. This also occurred when testing the algorithm on single pulse events, and is most likely due to the amount of variability in the pulse shapes. In such cases, it was assumed that the data resulted from a single pulse and the time of arrival, $t$, was in between the two consecutive time stamps, $t_{1}$ and $t_{2}$, respectively, and was calculated as follows

$$
t=t_{1}+\frac{E_{2}}{E_{1}+E_{2}}\left(t_{2}-t_{1}\right)
$$

The amplitude of the pulse, and any other pulse in the pileup, was obtained using least squares, as before. Using a shifted pulse led to a lower residual error in the reconstructed pileup signal than using consecutive, lower amplitude, pulses.

To assess the performance of the deconvolution algorithm on realistic data, we simulated pulse pileup events by shifting a single x-ray photon detector signal by a random amount, between $1 \mathrm{~ns}$ and $10 \mathrm{~ns}$, and adding it to another, unshifted, single $\mathrm{x}$-ray photon signal. For such simulated signal we know the exact times of arrivals and the pulse amplitudes, which are not known for real scatter detector signal, which captures some of the features of the real signal. The pulse pileup deconvolution algorithm was applied to 25050 simulated pulse pileup events. An error of $0.42 \pm 0.026 \mathrm{~ns}$, or $6.3 \pm 0.04 \mathrm{~cm}$ (in Compton scatter interaction location), Full Width at Half Maximum (FWHM) was achieved when comparing the reconstructed position and the shifted position. Figure 9 shows the distribution of errors between the reconstructed and the shifted $\mathrm{x}$-ray photon arrival time. The noise in the simulated pulse pileups was higher than in real pulse pileups, since two 


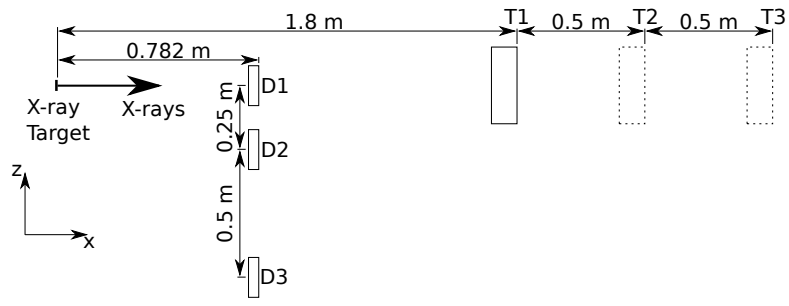

(a)

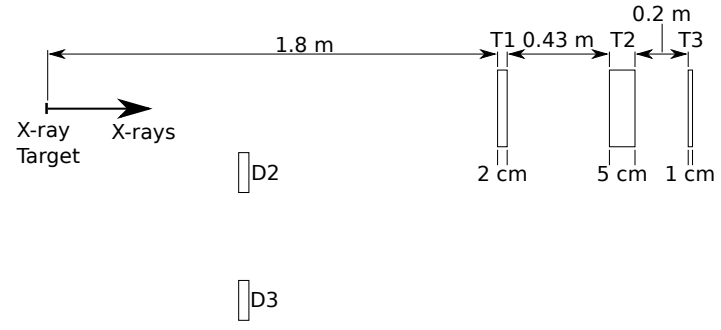

(b)

Fig. 6. a) A drawing of the geometry for experiment 1 (not to scale). A $5 \mathrm{~cm}$ thick plastic block was moved away from the source in steps of $50 \mathrm{~cm}$ (marked with dotted rectangles). Three detector positions were used (D1, D2, and D3), and three test object positions were used (T1, T2, and T3). For position T2, measurements were collected for all 3 detector positions, and for T1 and T3 measurements were collected at D1 only. The detector was positioned $44.5 \mathrm{~cm}$ below $(y=-44.5 \mathrm{~cm})$ the $\mathrm{x}$-ray beam at each position. The scatter for each object and detector position pair was measured separately. b) In Experiment 2 we measured the ToF of photons scattering from three plastic blocks places in series of thicknesses $2 \mathrm{~cm}, 5 \mathrm{~cm}$ and $1 \mathrm{~cm}$, separated by gaps of $43 \mathrm{~cm}$ and $20 \mathrm{~cm}$ looking from the source. The width and height of all the blocks were $15 \mathrm{~cm}$ and $25 \mathrm{~cm}$. Measurements were taken at detector positions D2 and D3.

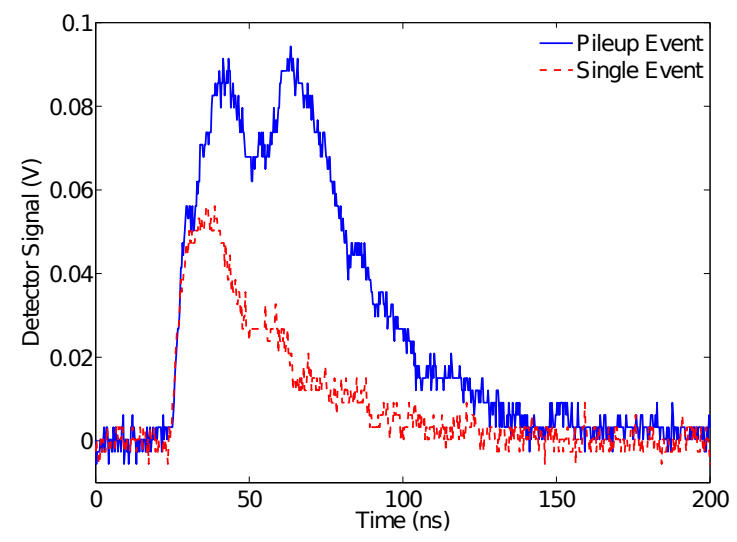

Fig. 7. A pulse pileup detector signal (solid line) and a single pulse detector signal (dashed red line). The deconvolution method returned 7 single pulses in the pileup event.

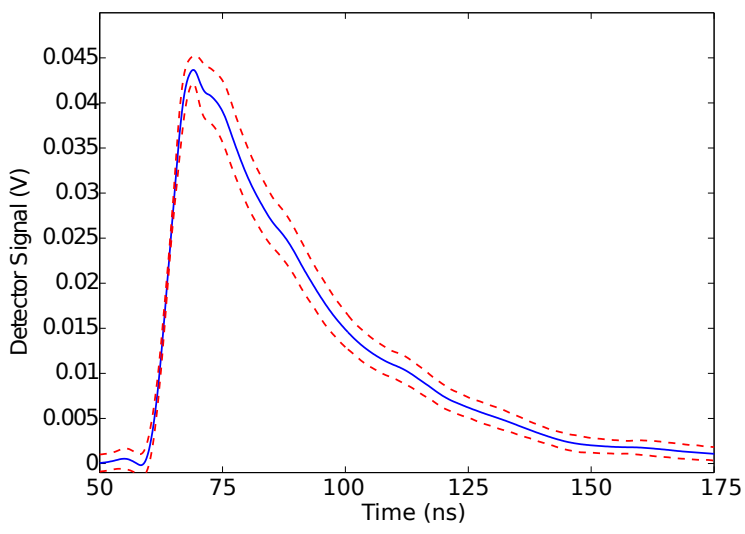

Fig. 8. The average pulse shape (solid line) and standard deviation (dashed line) for $45 \mathrm{mV}$ amplitude pulses.

noisy pulses were added together. Due to the overestimation of noise, the accuracy of the deconvolution method is expected to be slightly better than that shown in Figure 9.

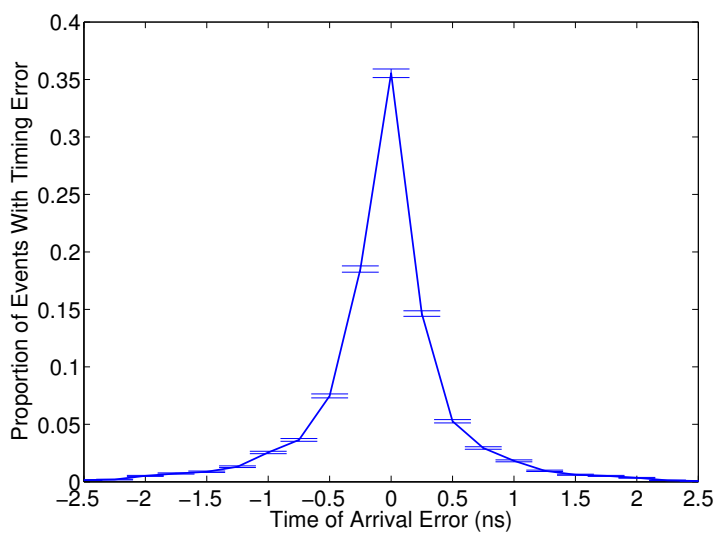

Fig. 9. The simulated error resulting from pulse pileup deconvolution.

\section{IDEAL RESOLUTION}

The calculation of the scatter points in Equation 3 assumes a point detector and a line source. The detector used had a diameter of $76 \mathrm{~mm}$ and the beam opening angle was $2^{\circ}$ which introduces an error into the reconstruction of the scattering point. This error was estimated using a Monte Carlo method. The x-ray source at VELA was simulated using GEANT4, a toolbox for the simulation of particles through matter [34]. The simulated electron beam, with a Gaussian energy distribution, mean energy of $4.55 \mathrm{MeV}$, and FWHM of $5 \%$, was incident upon the $\mathrm{x}$-ray target designed for the VELA experiments. The resulting $\mathrm{x}$-ray energy spectrum was used to simulate the ToF experiments. The $\mathrm{x}$-ray beam was modelled as a diverging pencil beam exiting the collimator aperture only, reducing the computation time required for the simulations. The pencil beam had a diameter of $1.1 \mathrm{~cm}$ at $30 \mathrm{~cm}$ from the target, the same diameter as the source collimator aperture. The scattered x-ray photons were recorded when they were incident upon the front face of the detector. Energy deposition and depth of interaction were not accounted for in the detector. $200,000,000$ photons were fired at the test objects in the simulation.

Table I shows the FWHM of calculated scatter positions due to the beam and detector size, for the different test object 


\begin{tabular}{|c||c|c|c|}
\hline & T1 & T2 & T3 \\
\hline D1 & $1.7 \pm 0.01 \mathrm{~cm}$ & $1.2 \pm 0.01 \mathrm{~cm}$ & $1.3 \pm 0.02 \mathrm{~cm}$ \\
D2 & n/a & $1.7 \pm 0.02 \mathrm{~cm}$ & n/a \\
D3 & n/a & $2.4 \pm 0.03 \mathrm{~cm}$ & n/a \\
\hline \multicolumn{3}{|c|}{ TABLE 1}
\end{tabular}

FWHM of Reconstructed Scatter Positions For Monte CARLO SIMULATION OF EXPERIMENT 1.

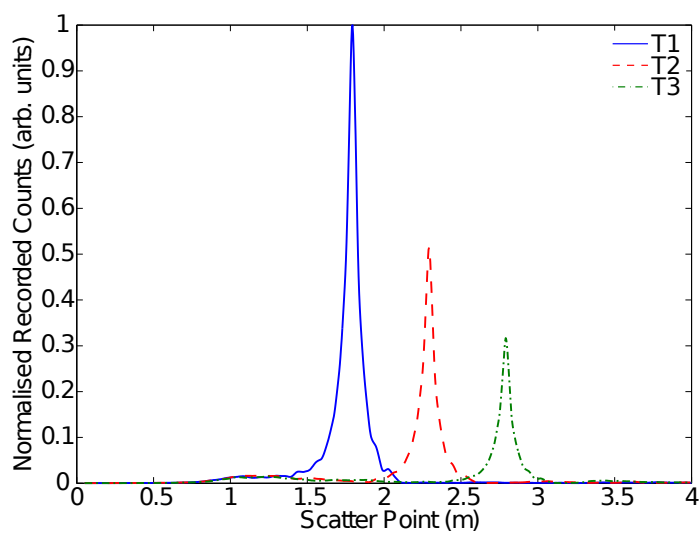

Fig. 10. Simulated scatter histogram for D1, with test objects at T1 (solid line), T2 (dashed line), and T3 (dash-dot line), with deconvolution error applied.

\begin{tabular}{|c||c|c|c|}
\hline & T1 & T2 & T3 \\
\hline D1 & $7.7 \pm 0.06 \mathrm{~cm}$ & $7.5 \pm 0.08 \mathrm{~cm}$ & $7.4 \pm 0.1 \mathrm{~cm}$ \\
D2 & n/a & $7.7 \pm 0.08 \mathrm{~cm}$ & n/a \\
D3 & n/a & $8.2 \pm 0.1 \mathrm{~cm}$ & n/a \\
\hline
\end{tabular}

FWHM OF RECONSTRUCTED SCATTER POSITIONS FOR THE MONTE CARLO SIMULATION OF EXPERIMENT 1 ACCOUNTING FOR THE ERROR OF THE DECONVOLUTION ALGORITHM.

placements and detector positions. As the test object is moved further back, although the beam size increases, the solid angle presented by the detector decreases and the time spread also decreases. When the test object was placed at T3 the FWHM increased when compared to T2. It is not known whether this is because the size of the beam starts to dominate the spread of the ToF of the $\mathrm{x}$-ray photons, or whether there is another geometrical effect taking place.

Although the test object is $5 \mathrm{~cm}$ thick, attenuation in the test object reduces the number of detected $\mathrm{x}$-ray photons that scatter at the back of the test object, reducing the simulated FWHM. This effect should be constant at each test object position.

The uncertainty in ToF due to the pulse deconvolution algorithm was introduced into the simulation by convolving the simulated scatter histogram with the ToF error from the distribution given in Figure 9, emulating the spatial resolution achievable by an ideal detector with no background present. The result is depicted in Figure 10, for detector in position D1 (the counts have been normalised to the maximum counts recorded at T1). The corresponding FWHM for all three detector positions are summarised in Table II.

To account for finite timing resolution of the detector, we introduced an extra ToF error drawn from a Gaussian distribution. Therefore, the simulated scatter histograms were

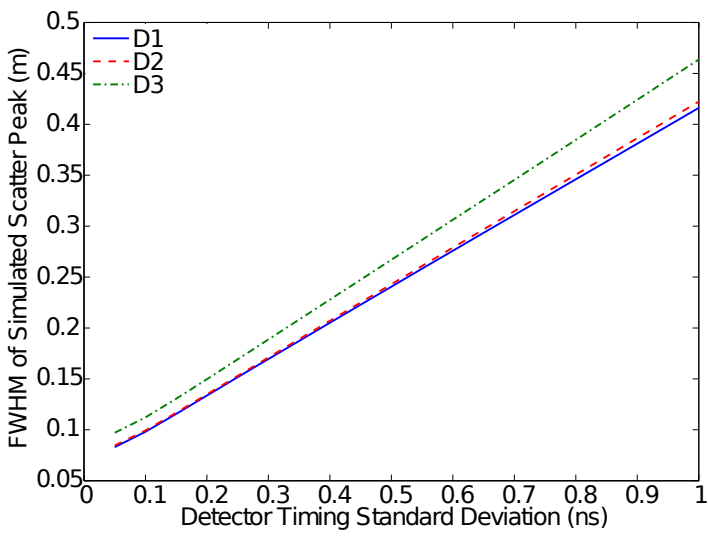

Fig. 11. The effect of detector timing resolution on the FWHM of the simulated scatter peaks for D1 (solid line), D2 (dashed line), and D3 (dash-dot line) for the test object position $\mathrm{T} 2$.

convolved with Gaussian detector timing error distribution prior to convolution with the distribution of the error of the deconvolution algorithm. Figure 11 shows a plot of the FWHM of the simulated scatter peaks against the standard deviation of the detector timing resolution for the three detector positions and the object in position $\mathrm{T} 2$. The standard deviation of the detector timing resolution was varied from $50 \mathrm{ps}$ to $1 \mathrm{~ns}$ in 50 ps steps. At detector position D1, the FWHM varied from $8.3 \mathrm{~cm}$ to $41.8 \mathrm{~cm}$ when the test object was positioned at T1, and from $8.1 \mathrm{~cm}$ to $41.1 \mathrm{~cm}$ when positioned at T3. The timing resolution of the detector was measured to be $443 \pm 7 \mathrm{ps}$ at $511 \mathrm{keV}$ using a Na-22 source, which extrapolates to a value of $846 \pm 13 \mathrm{ps}$ at $140 \mathrm{keV}$, the mean energy of the scattered photons in simulation, assuming the resolution is dominated by Poisson statistics.

\section{EXPERIMENTAL RESULTS}

\section{A. Background Measurements}

The recorded ToF of the $\mathrm{x}$-ray photons were converted to scattering positions, assuming a true pencil beam, using Equations 3,4. The scattering positions were then histogrammed with bin size $3.76 \mathrm{~cm}$ determined by the sampling rate of the oscilloscope, ( $3.76 \mathrm{~cm}$ corresponds to 250 ps photon travel time). Figure 12 shows the scatter position histogram for detector position D1 with no test object present. Due to the delay in the cables and the unknown temporal offset between the electron beam arrival and the trigger pulse, the time of creation of the $\mathrm{x}$ rays, i.e. $t=0$, was unknown and hence it had to be estimated from ToF of primary photons (photons which travel through the collimator shielding straight into the detector). Those photons cause the initial sharp peak in Figure 12, and their arrival at the detector corresponds to $t=3 \mathrm{~ns}$.

The peak around $7.5 \mathrm{~m}$ in Figure 12 was due to $\mathrm{x}$-ray photons in the primary beam scattering off the back wall of the user area, which was located approximately $7.5 \mathrm{~m}$ away from the target.

Background subtraction was performed on all scatter histograms. To this end the background histogram was first 


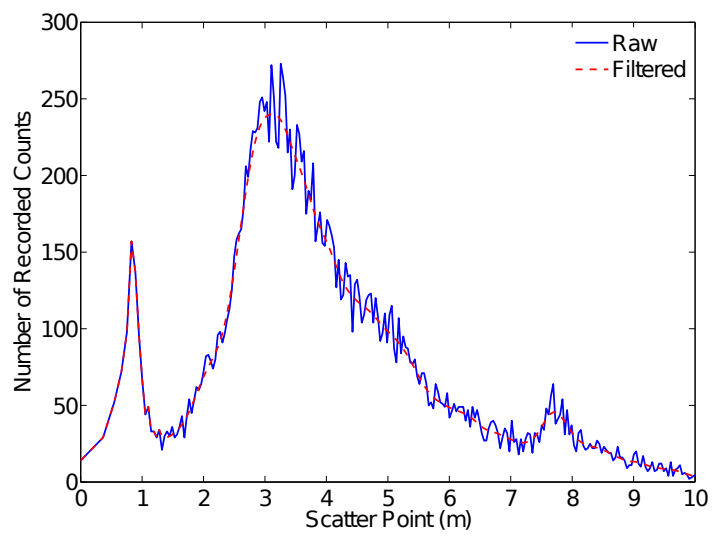

Fig. 12. Raw (solid line) and filtered (dashed line) scatter histogram for detector Position D1, with no test object present. The large signal is due to $\mathrm{X}$-ray production in the unshielded beam pipe, upstream of the target, caused by a large beam size at VELA. These x-ray photons scattered in the room before detection.

filtered with Blackman windowed sinc filter with a length of 33 points and cutoff frequency of $0.125 \pi \mathrm{rad} / \mathrm{s}$, and then scaled. Applying the filter leads to reduction in amplitude and increase in width of the sharp initial peak at $0.9 \mathrm{~m}$ in the background histogram and would result in large errors in background subtraction. Therefore, the filter was applied to the histogram values after $1.3 \mathrm{~m}$ and this initial peak was left unfiltered. Figure 12 shows a plot of the filtered background obtained at detector position D1. We found that the most effective scaling was using the ratio of the total counts in the background and the test object histograms between $3.5 \mathrm{~m}$ and $4.0 \mathrm{~m}$. This signal was due to photons produced in the beam pipe and gives a good estimation of the ratio of the bunch charges.

The high background present contributed a significant level of noise to the ToF scatter histograms. A separate data acquisition was performed with the detector a further $250 \mathrm{~mm}$ to the left of D3, so that it had direct line of sight to the unshielded beam pipe. During this acquisition the detector was saturated due to the large number of incident $\mathrm{x}$-ray photons, suggesting that the electron beam was interacting with the beam pipe, creating the large background signal present.

The scaled filtered background is different than the background present during the measurement with one object at position T1, as shown in Figure 13. It is lower between $2 \mathrm{~m}$ and $3 \mathrm{~m}$ while higher between $3 \mathrm{~m}$ and $4.25 \mathrm{~m}$. When the test object was moved to position T2, the filtered scaled background shows a better correlation, as depicted in Figure 14 though it still is slightly lower than the background in the object signal. These differences are due to the fluctuations in the beam.

\section{B. Experiment 1}

The background subtracted histograms for the object positioned at $\mathrm{T} 1, \mathrm{~T} 2$, and $\mathrm{T} 3$, respectively, and the detector at $\mathrm{D} 1$ have been plotted in Figure 15.

Figure 15 shows the peak scatter intensity occurring in the histogram bins centred at $1.81 \mathrm{~m}, 2.31 \mathrm{~m}$, and $2.84 \mathrm{~m}$, and the

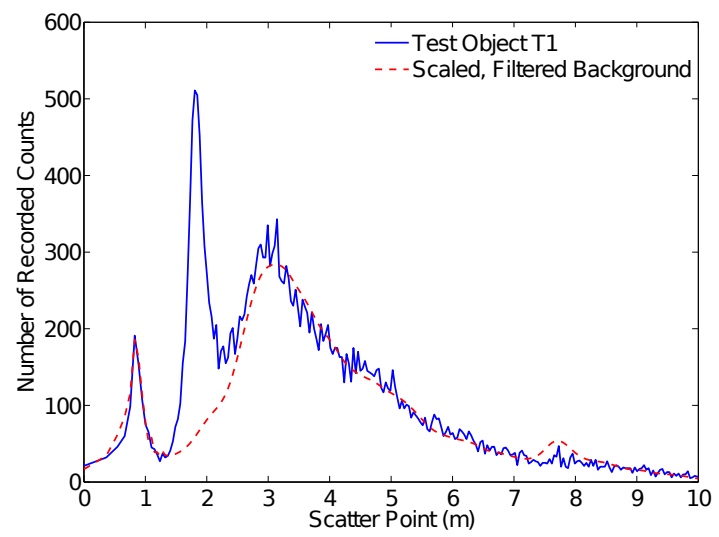

Fig. 13. ToF scatter histogram for the test object positioned at T1 (solid line) and the scaled background histogram (dashed line) for D1.

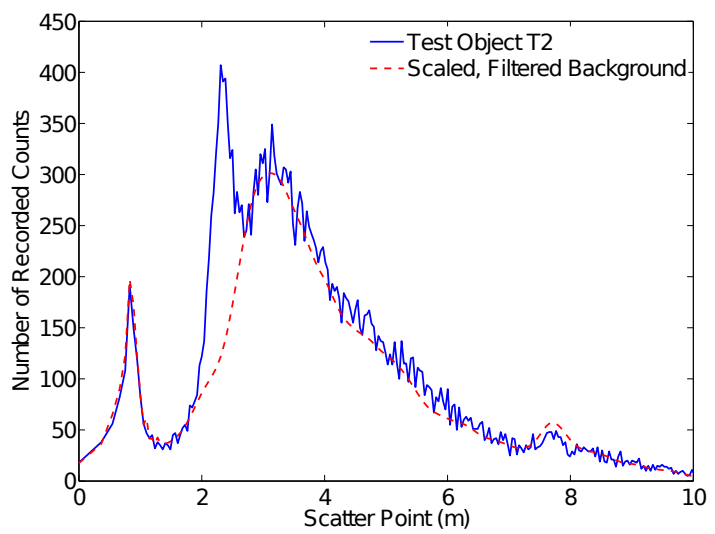

Fig. 14. ToF scatter histogram for the test object positioned at T2 (solid line) and the scaled background histogram (dashed line) for D1.

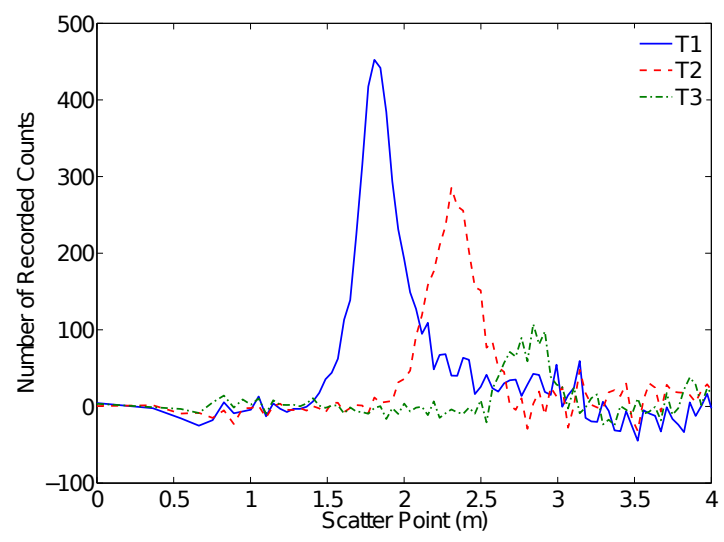

Fig. 15. Scatter histogram for the detector at D1, with test objects at $\mathrm{T} 1$ (solid line), T2 (dashed line), and T3 (dash-dot line).

FWHM for the three positions were $29 \pm 0.46 \mathrm{~cm}, 36 \pm 0.70 \mathrm{~cm}$, and $29 \pm 1.04 \mathrm{~cm}$, respectively. The initial peak, located at $0.9 \mathrm{~m}$, is still present in the histograms. The background subtraction leads to some parts of the histograms showing negative counts. This is caused by the electron beam moving, 


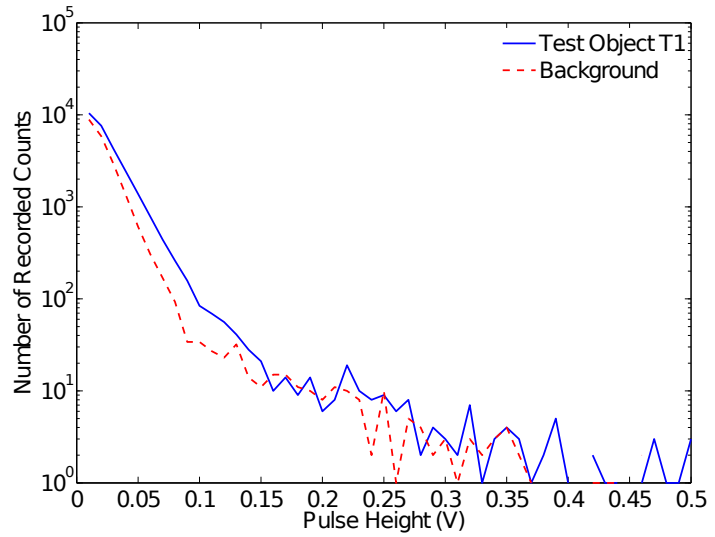

Fig. 16. The measured pulse height spectra of the scattered $x$-ray photons when no test object was in place (dashed line) and when a $5 \mathrm{~cm}$ test object was placed at T1 (solid line), recorded at D1. The energy of photons with a $60 \mathrm{mV}$ pulse height was approximately $200 \mathrm{keV}$.

altering the background scatter distribution, in between data collection runs. This was particularly visible when the test object was positioned at T1. The movement of the electron beam lead to a high background count between $2.25 \mathrm{~m}$ and $3.25 \mathrm{~m}$, visible in Figure 15, even after background subtraction.

The FWHM can be reduced by filtering out the lowenergy $\mathrm{x}$-ray photons in the scatter histograms. This is because scintillation is described by Poisson statistics, and the timing resolution of a scintillator is proportional to the number of optical photons created by an x-ray photon, as well as the decay time of the scintillator. Low-energy deposition in the detector leads to fewer optical photons and poorer timing resolution. Low-energy $\mathrm{x}$-ray photons will also scatter off the surface of the test object and high-energy photons will penetrate deeper before scattering. By filtering out the lowenergy photons the scatter position histogram should become less foward peaked. For detector position D1, a cut off level of $60 \mathrm{mV}$, approximately $200 \mathrm{keV}$, was found to provide the optimal balance between full width at half maximum and number of counts. Figure 16 shows the recorded pulse height spectra of the detected $\mathrm{x}$-ray photons at D1, and Figure 17 shows the effect of applying a $60 \mathrm{mV}$ filter to the scatter histograms with the test object positioned at $\mathrm{T} 1$ and $\mathrm{T} 2$. Background subtraction was performed by using the same low-energy filter, and then subtracting as described previously. The energy filtering resulted in a reduction of FWHM was $12 \pm 0.72 \mathrm{~cm}$ and $17 \pm 0.98 \mathrm{~cm}$ for $\mathrm{T} 1$ and $\mathrm{T} 2$, respectively. The large negative counts in Figure 17 between $0 \mathrm{~m}$ and $1.3 \mathrm{~m}$ was caused by the higher energy of the photons detected during the background measurement. This might have been due to variations in the electron beam between measurements. For the object position $\mathrm{T} 3$, the scattered photons have energies below the cut-off and hence the low-energy filtering is not applicable and the histogram is omitted from the plot.

Figure 18 shows a plot of the scatter histograms for test object positioned at $\mathrm{T} 2$ for the three detector positions, with a $60 \mathrm{mV}$ cut off applied. The FWHMs were $17 \pm 0.72 \mathrm{~cm}, 18 \pm$

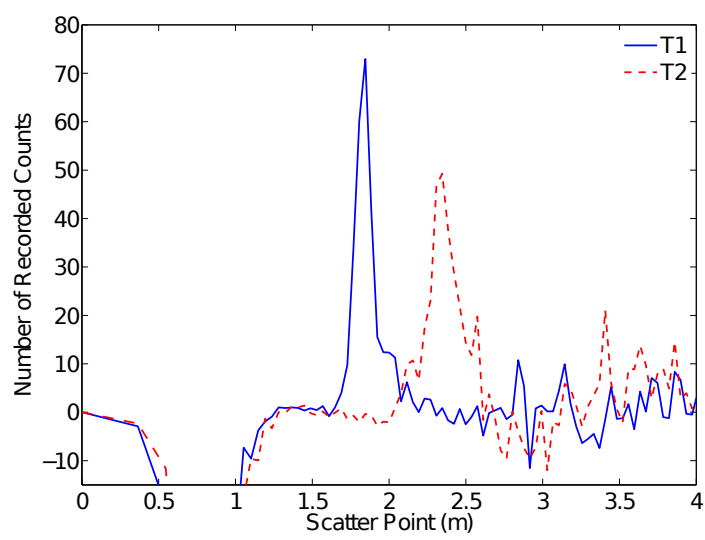

Fig. 17. The effect of filtering out low-energy photons on the D1 scatter histograms, for test objects at $\mathrm{T} 1$ (solid line) and $\mathrm{T} 2 \mathrm{~m}$ (dashed line).

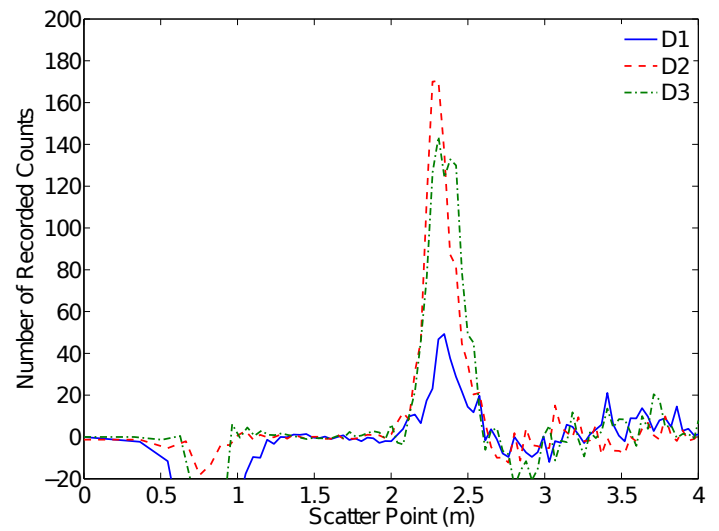

Fig. 18. Scatter histogram for the test object positioned at T2 for D1 (solid line), D2 (dashed line), and D3 (dash-dot line).

$0.58 \mathrm{~cm}$, and $22 \pm 0.70 \mathrm{~cm}$ for D1, D2, and D3, respectively, which exhibits the same general trend as seen in the Monte Carlo simulations in Table II.

\section{Experiment 2}

The three plastic blocks in sequence were inseparable without filtering out the low-energy photons. The corresponding raw background subtracted ToF histograms for positions D2 and D3 are shown in Figure 19. It is not clear from these histograms that there are several test objects under inspection, without a priori knowledge.

By applying the low-energy filter, as before, the front two test objects become separated in the histogram and are visible as two peaks. It was found that a cut off level of $70 \mathrm{mV}$ was optimal for separating the data from the individual test objects. Figure 20 presents a plot of the ToF histograms in Figure 19 with a filter of $70 \mathrm{mV}$ applied. For D2, the first peak has a FWHM of $17 \pm 0.70 \mathrm{~cm}$, and the second peak has a FWHM of $26 \pm 1.26 \mathrm{~cm}$. For D3, the first peak has a FWHM of $15 \pm 1.28 \mathrm{~cm}$, and the second peak has a FWHM of $27 \pm 1.08 \mathrm{~cm}$.

The third plastic block is not visible as a separate peak in the low-energy filtered histograms due to low scatter flux which 


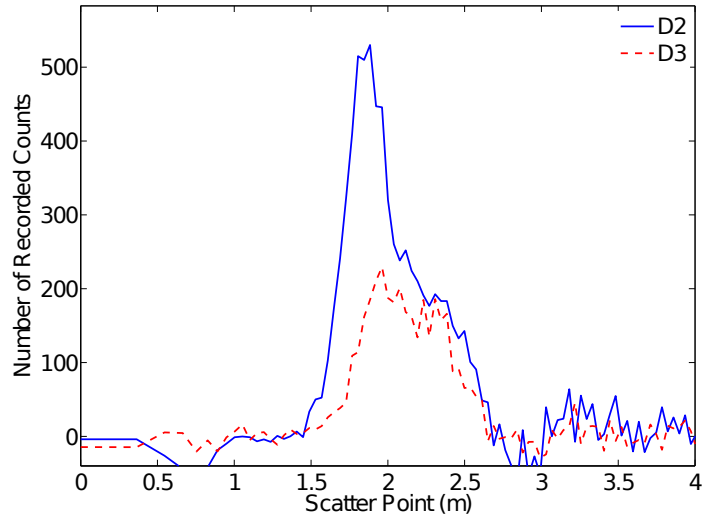

Fig. 19. ToF scatter histogram for three test objects in series for D2 (solid line) and D3 (dashed line).

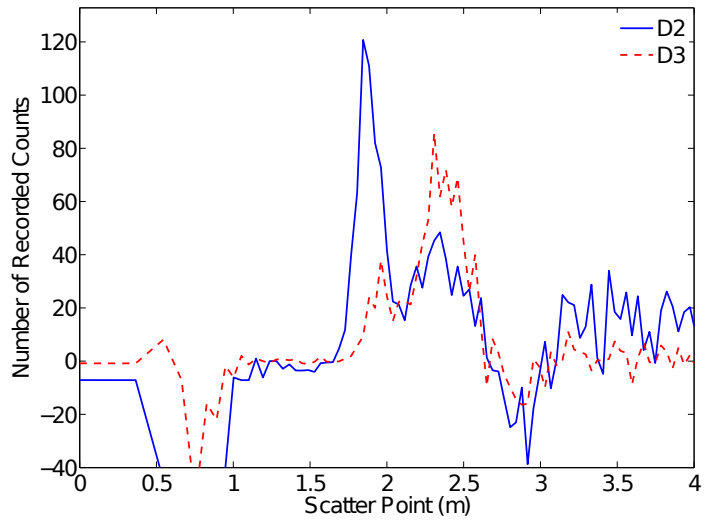

Fig. 20. ToF scatter histogram for three test objects in series for D2 (solid line) and D3 (dashed line), with a $70 \mathrm{mV}$ cut off applied.

we attribute to the block thickness of only $1 \mathrm{~cm}$, attenuation of the incident photons through the two other blocks as well as to it being positioned relatively close, only $20 \mathrm{~cm}$ apart, from the second block.

\section{DISCUSSION}

The experimental results matched the general trend seen in the simulation results; as the source to test object distance was increased, the FWHM of the scatter signal also increased. Moreover, the FWHM of the scatter signal also increased with increasing test object to detector distance. The experimental results in Figure 18 suggest a detector timing resolution of between 300 and $500 \mathrm{ps}$, when compared with the results in Figure 11, at the cut off voltage of $60 \mathrm{mV}$ which was approximately equal to $200 \mathrm{keV}$ deposited energy. This value is lower than the measured timing resolution of the detector.

When three test objects were placed in series, the measured signals resulting from the front two objects became separated by an air gap in the histogram by applying a low-energy cut off. The lack of separation of low-energy scattered photons could be attributed to poor timing resolution of the detector at lower energies. Another possibility is multiple scatter from the objects and their holder which would also give rise to a lowenergy signal between the two front objects. In simulation, it was estimated that $53.5 \%$ of photons detected at $D 2$ were single scatters, $31.6 \%$ of detected photons scattered multiple times in a single object, and $14.9 \%$ of detected photons scattered in multiple objects. At D3, these values changed to $49.1 \%, 31.8 \%$, and $19.0 \%$. The error in each case was less than $10^{-4 \%}$.

As shown in Figures 13 and 14, the background was not constant during the experiments. Due to background fluctuations, background subtraction was not possible to a high accuracy and the measured FWHMs of the histograms might have been altered (broadened) as a result. It is believed that the background also varied in energy. The large negative counts between $0 \mathrm{~m}$ and $1.3 \mathrm{~m}$ in Figure 20 are due to the higher energy of the primary photons escaping the shielding directly into the detector. This suggests some energy fluctuation in the beam, or inaccuracy of the amplitudes of the deconvolved pulses.

The accuracy of the ToF method was also affected by the high standard deviation on the falling edge of the pulses, as seen in Figure 8. The time of arrival of pulses occurring on the falling edge of another pulse contained greater uncertainty than two pulses occurring close together. Some of this uncertainty may have been reduced by increasing the voltage of the detector. It can be seen in Figure 7 that the vertical scale on oscilloscope was set too large $( \pm 0.7 \mathrm{~V})$, and although the resolution was 8-bit, the full vertical range was not used, reducing the number of effective bits. For example $99 \%$ of the pulses recorded from the background signal at detector Position 1 had a pulse height of $120 \mathrm{mV}$ or less. This led to large quantisation error in the signal, decreasing the accuracy of the pulse pileup deconvolution method.

Our idealised resolution estimation method does not take into account factors like large scatter background or the quantisation error, while it most likely underestimates the accuracy of the pulse deconvolution algorithm due to higher level of noise resulting from summation of multiple noisy pulses. Nonetheless, the latter effect is small, thus we believe that the estimate is close to the best attainable resolution given geometry, detector characteristic and accuracy of deconvolution algorithm. The Monte Carlo simulations suggest the estimated timing resolution of the detector is between 300 and $500 \mathrm{ps}$, however the large variation seen in the experimentally measured FWHM is most likely due to the background subtraction errors and low number of recorded counts. The timing resolution at $200 \mathrm{keV}$, the cut off energy used, was estimated at 708 ps using extrapolation from the measured value. The discrepancy between the simulation and the experimentally measured value could be due to the high background and the difficulties associated with background subtraction. The simulated pulse pileup events also had double noise, which may have increased the estimated error of the deconvolution method and lead to inaccuracies in Figure 11.

The simulations suggest that both the detector timing resolution and pulse deconvolution accuracy dominate the error of ToF CSI. Detector timing resolution is fixed by the equipment, although improving at a fast rate, and efforts should be made 
to increase the accuracy of the pulse pileup deconvolution method. Although turning down the beam current would result in fewer pulse pileups, this was not possible during these measurements as it lead to large fluctuations in the beam position and current.

\section{CONCLUSION}

The present research describes the first attempt to use ToF to reconstruct the position of scatter of $\mathrm{x}$-ray photons. Experiments have been performed at VELA, achieving an accuracy of the order of tens of centimetres, in the presence of a high radiation background caused by the suboptimal electron beam and shielding. It is expected that, were the experiments to be repeated, the spatial resolution would be improved by shielding the beam pipe and having a more stable beam. The background subtraction would be much simpler in such a situation, and the associated problems would be removed. Despite the large background, test objects were visible in the scatter histograms, and test objects in series were separated after applying a low energy cut off. The results suggest that ToF can lead to the reconstruction of scatter points to provide three dimensional information of the object under inspection. The results also suggest that the technique will not be able to resolve fine details, and should be aimed at creating a three dimensional image with a relatively low number of slices in the dimension along the photon path. Due to the high energies used, it might be beneficial to combine the method with transmission imaging and simplify the image analysis, as a beam stop will be required for shielding.

\section{ACKNOWLEDGMENT}

\section{REFERENCES}

[1] E. J. Morton, K. Mann, A. Berman, M. Knaup, and M. Kachelreiss, "Ultrafast 3D reconstruction for X-ray real-time tomography (RTT)," in IEEE Nuclear Science Symposium Conference Record, 2009, pp. 40774080 .

[2] C. Reid, M. Betcke, D. Chana, and R. Speller, "The development of a pseudo-3D imaging system (tomosynthesis) for security screening of passenger baggage," Nuclear Instruments and Methods in Physics Research A Accelerators Spectrometers Detectors and Associated Equipment, vol. 652, no. 1, pp. 108-111, Oct 2011.

[3] G. Zentai, "X-ray Imaging for Homeland Security," in IEEE International Workshop on Imaging Systems and Techniques, Sep. 2008, pp. $1-6$.

[4] N. V. Arendtsz and E. M. A. Hussein, "Energy-spectral Compton scatter imaging-Part I: Theory and mathematics," IEEE Trans. Nucl. Sci., vol. 42, no. 6, pp. 2155-2165, Dec. 1995.

[5] P. J. Arsenault and E. M. A. Hussein, "Image Reconstruction from the Compton Scattering of X-Ray Fan Beams in Thick / Dense Objects," IEEE Trans. Nucl. Sci., vol. 53, no. 3, pp. 1622-1633, 2006.

[6] F. A. Smith, A Primer in Applied Radiation Physics. World Publishing Co. Pte. Ltd, 2000.

[7] M. Conti, "Focus on time-of-flight PET: the benefits of improved time resolution," Eur J Nucl Med Mol Imaging, vol. 38, pp. 1147-1157, dec 2010.

[8] V. C. Spanoudaki and C. S. Levin, "Photo-detectors for Time of Flight Positron Emission Tomography (ToF-PET)." Sensors, vol. 10, pp. 10484-10 505, Jan. 2010.

[9] M. Conti, L. Eriksson, H. Rothfuss, and C. Melcher, "Comparison of Fast Scintillators With TOF PET Potential," IEEE Trans. Nucl. Sci., vol. 56, no. 3, pp. 926-933, jun 2009.

[10] T. Frach, G. Prescher, C. Degenhardt, R. de Gruyter, A. Schmitz, and R. Ballizany, "The digital silicon photomultiplier - Principle of operation and intrinsic detector performance," in Nuclear Science Symposium Conference Record (NSS/MIC) 2009. IEEE, Oct 2009, pp. 1959-1965.
[11] L. H. Braga, L. Gasparini, L. Grant, R. K. Henderson, N. Massari, M. Perenzoni, D. Stoppa, and R. Walker, "Complete characterization of SPADnet-I - a digital 816 SiPM array for PET applications," in Nuclear Science Symposium and Medical Imaging Conference Record (NSS/MIC), 2013. IEEE, Oct 2013, pp. 1-4.

[12] D. R. Schaart, S. Seifert, R. Vinke, H. T. van Dam, P. Dendooven, H. Löhner, and F. J. Beekman, " $\mathrm{LaBr}_{3}$ :Ce and SiPMs for time-of-flight PET: achieving 100 ps coincidence resolving time," Physics in Medicine and Biology, vol. 55, pp. N179-N189, jan 2010.

[13] M. Moszyński, M. Gierlik, M. Kapusta, A. Nassalski, T. Szczęśniak, C. Fontaine, and P. Lavoute, "New Photonis XP20D0 photomultiplier for fast timing in nuclear medicine," Nuclear Instruments and Methods in Physics Research A, vol. 567, pp. 31-35, jun 2006.

[14] J. Kostamovaara and M. Karras, "Distance determination by the gammaray time-of-flight method," IEEE Transactions on Instrumentation and Measurement, vol. 41, pp. 616-621, Oct 1992.

[15] J. Tickner, M. Currie, and G. Roach, "Feasibility study for a low-cost 3D gamma-ray camera," Applied Radiation and Isotopes, vol. 61, pp. 67-71, 2004.

[16] C. Ferguson and D. Ramsden, "The feasibility of searching for contraband materials using a gamma-ray 'radar' system," in Nuclear Science Symposium Conference Record, 2004. IEEE, Oct 2004, pp. 1961-1965.

[17] L. Gurdev, T. Dreischuh, D. Stoyanov, and Protochristov, "On the Possibility of Determining the Distribution of a Substance Penetrating into a Dense Medium Using Gamma-Ray Detection and Ranging Approach," Acta physica Polonica. A, vol. 118, no. 4, pp. 540-549, 2010.

[18] D. Lee, M. Cowee, E. Fenimore, M. Galassi, Q. Looker, W. VoganMcNeil, L. Stonehill, and M. Wallace, "Three-Dimensional Imaging of Hidden Objects Using Positron Emission Backscatter," in IEEE Nuclear Science Symposium Conference Record 2009. IEEE, Oct 2009, pp. 1894-1896.

[19] Q. Looker, L. Stonehill, M. Wallace, M. Galassi, M. Cowee, E. Fenimore, and W. Vogan-McNeil, "Demonstration of imaging via backscattering of annihilation gamma rays," Nuclear Instruments and Methods in Physics Research A, vol. 615, pp. 295-300, 2010.

[20] S. Seifert, H. T. van Dam, and D. R. Schaart, "The lower bound on the timing resolution of scintillation detectors," Physics in Medicine and Biology, vol. 57, no. 7, pp. 1797-1814, Apr. 2012.

[21] P. Scoullar, C. McLean, and R. Evans, "Real time pulse pileup recovery in a high throughput digital pulse processor," in AIP Conference Proceedings, vol. 1412, 2011, pp. 271-277.

[22] A. Wang, D. Harrison, V. Lobastov, and J. Tkaczyk, "Pulse pileup statistics for energy discriminating photon counting x-ray detectors," Medical Physics, vol. 38, no. 7, pp. 4265-4275, 2011.

[23] M. Bolic, V. Drndarevic, and W. Gueaieb, "Pileup Correction Algorithms for Very-High-Count-Rate Gamma-Ray Spectrometry With NaI(Tl) Detectors," IEEE Transactions on Instrumentation and Measurement, vol. 59, no. 1, pp. 122-130, jan 2010.

[24] Z. Deng, Q. Xie, Z. Duan, and P. Xiao, "Scintillation event energy measurement via a pulse model based iterative deconvolution method," Physics in Medicine and Biology, vol. 58, pp. 7815-7827, 2013.

[25] R. Novak, J. Stefan, and M. Vencelj, "Gauss-Seidel Iterative Method as a Real-Time Pile-Up Solver of Scintillation Pulses," IEEE Trans. Nucl. Sci., vol. 56, no. 6, pp. 3680-3687, dec 2009.

[26] L. Gurdev, T. Dreischuh, and D. Stoyanov, "Deconvolution of LongPulse Lidar Profiles," in Lasers - Applications in Science and Industry, K. Jakubczak, Ed. InTech, Dec. 2011, ch. 13, pp. 249-276.

[27] P. A. McIntosh et al., "Implementation and commissioning of the new versatile electron linear accelerator (formerly EBTF) at Daresbury Laboratory for industrial accelerator system development," in Proceedings of IPAC2013, 2013, pp. 3708-3710.

[28] B. L. Militsyn et al., "Photoinjector of the EBTF/CLARA facility at Daresbury," in Proceedings of LINAC2012, 2012.

[29] J. W. McKenzie, D. Angal-Kalinin, J. K. Jones, and B. L. Militsyn, "VELA (formerly EBTF) simulations and first beam commissioning," in Proceedings of IPAC2013, 2013, pp. 431-433.

[30] J. Glodo, A. Kuhn, W. M. Higgins, E. V. D. Van Loef, J. S. Karp, W. M. Moses, S. E. Dorenze, and K. S. Shah, "CeBr 3 for Time-ofFlight PET," in IEEE Nuclear Science Symposium Conference Record, 2006, pp. 1570-1573.

[31] S. E. Derenzo, W.-S. Choong, and W. W. Moses, "Fundamental limits of scintillation detector timing precision," Physics in Medicine and Biology, vol. 59, pp. 3261-3286, 2014.

[32] G. F. Knoll, Radiation Detection and Measurement, 4th ed. John Wiley \& Sons, Inc, 2010.

[33] National Instruments Corporation, 11500 North Mopac Expressway, Austin, TX, 78759-3504, http://www.ni.com/labview. 
[34] S. Agostinelli et al., "Geant4-A Simulation Toolkit," Nuclear Instruments and Methods in Physics Research A, vol. 506, no. 3, pp. 250-303, 2003. 